\title{
The Non-associative Lambek calculus with product in polynomial time
}

\author{
Philippe de Groote \\ LORIA UMR $\mathrm{n}^{\circ} 7503$ - INRIA \\ Campus Scientifique, B.P. 239 \\ 54506 Vandœuvre lès Nancy Cedex - France \\ e-mail: degroote@loria.fr
}

\begin{abstract}
We prove, by introducing a new kind of sequent calculus, that the decision problem for the non-associative Lambek calculus with product belongs to PTIME. This solves an open prolem.
\end{abstract}

\section{Introduction}

Modern categorial grammars [6] are based on a logical calculus introduced by Lambek more than thirty years ago $[4,5]$. Two variants of this calculus exist. The first, $\mathbf{L}$, which is perhaps the most well-known, corresponds exactly to the noncommutative fragment of IMLL, i.e., intuitionistic multiplicative linear logic [2]. The second, NL, which was introduced three years later, is obtained from the first by dropping the hidden structural rule of associativity. Therefore intuitionistic multiplicative linear logic may be seen as the commutative extension of $\mathbf{L}$ which, in turn, may be seen as the associative extension of NL:

$$
\text { NL } \subset \mathbf{L} \subset \mathbf{I M L L}
$$

If, in addition, we distinguish between the purely implicational fragments and the fragments with product, the picture becomes the following:

$$
\begin{aligned}
& \mathrm{NL}^{\backslash / \bullet} \subset \mathbf{L}^{\backslash / \bullet} \subset \mathbf{I M L L}^{-\circ \otimes}
\end{aligned}
$$

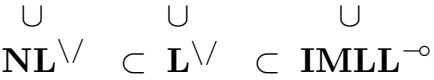

where the superscripts make explicit the connectives of the systems.

The decidability of these six fragments follows immediately from easy cut elimination theorems. As for the complexity of the associated decision problems, the state of the art is as follows. Kanovich has shown both $\mathbf{I M L L}^{-\circ \otimes}$ and IMLL ${ }^{-\circ}$ to be NP-complete [3]. ${ }^{1}$ In the case of $\mathbf{L}^{\backslash} / \bullet$ and $\mathbf{L}^{\backslash}$, the question is still open. Moreover, there is no proof that the two problems are equivalent. Aerts and Trautwein have shown that NL ${ }^{\bigvee}$ belongs to PTIME [1]. Our own contribution is to show that this is also the case for $\mathrm{NL}^{\backslash / \bullet}$.

\footnotetext{
${ }^{1}$ In fact, in this case, the two problems are easily seen to be equivalent by using a Goedel-like negative translation. This is not true for $\mathbf{L}$ and NL because Goedel-like translations do not work in a non-commutative setting.
} 


\section{The non-associative Lambek calculus}

The formulas of the non-associative Lambek calculus with product , NL $\backslash / \bullet$, are built from a set of atomic formulas $\mathcal{A}$ and the connectives $\backslash, /$, and $\bullet$ according to the following grammar:

$$
\mathcal{F}::=\mathcal{A}|(\mathcal{F} \backslash \mathcal{F})|(\mathcal{F} / \mathcal{F}) \mid(\mathcal{F} \bullet \mathcal{F})
$$

The consequence relation of $\mathbf{N L} \backslash / \bullet$ may be specified by a Gentzen-like sequent calculus. The sequents have the form $\Gamma \vdash A$ where $\Gamma$ is a non-empty binary tree of formulas, i.e., a fully bracketed structure. We take for granted the notion of context, i.e., a binary tree with a hole. If $\Gamma[]$ is such a context, $\Gamma[A]$ denotes the binary tree obtained by filling the hole in $\Gamma[]$ with the formula $A$.

$A \vdash A$

(Id)

$$
\begin{aligned}
& \frac{\Gamma \vdash A \quad \Delta[B] \vdash C}{\Delta[(\Gamma,(A \backslash B))] \vdash C} \\
& \frac{(A, \Gamma) \vdash B}{\Gamma \vdash(A \backslash B)} \\
& \frac{\Gamma \vdash A \quad \Delta[B] \vdash C}{\Delta[((B / A), \Gamma)] \vdash C} \\
& \frac{(\Gamma, A) \vdash B}{\Gamma \vdash(B / A)} \\
& \frac{\Gamma[(A, B)] \vdash C}{\Gamma[(A \bullet B)] \vdash C} \\
& \frac{\Gamma \vdash A \Delta \vdash B}{(\Gamma, \Delta) \vdash(A \bullet B)}
\end{aligned}
$$

The binary-tree structure of the antecedents induces the non-associativity of the calculus. As an illustration, consider the following derivation:

$$
\frac{a \vdash a \frac{b \vdash b \quad c \vdash c}{(b, b \backslash c) \vdash c}}{((a, a \backslash b), b \backslash c) \vdash c}
$$

In the associative case, this derivation might be continued by applying the right introduction rule of $\backslash$, which would yield $(a \backslash b, b \backslash c) \vdash a \backslash c$. In the present case, the bracketing of the antecedent prevents Rule $(\backslash-\mathrm{R})$ from being applied.

In order to show that one may decide in polynomial time whether a sequent of $\mathbf{N L} \backslash \bullet$ is derivable, we will focus on sequents made of two formulas. By doing so, we will not lose any generality, as explained below.

Proposition 1. Rule $\bullet-L$ is invertible.

Proof. This follows from the fact that this rule is permutable with all the rules.

From this, we immediately have:

Corollary 1. For each sequent $\Gamma \vdash B$ there exist a formula $A$ such that $\Gamma \vdash B$ is provable if and only if $A \vdash B$ is provable. Moreover, $\Gamma \vdash B$ and $A \vdash B$ have the same length. 
Because of Corollary 1, we may reduce the decision problem of $\mathbf{N L} \backslash \bullet$ to the particular case of sequents made of two formulas. Let us call any such provable sequent a tautology of $\mathbf{N L} \backslash \bullet \bullet$. We end this section by giving a characterisation of these tautologies.

Proposition 2. The set of tautologies of $\mathbf{N L} \backslash / \bullet$ is the least set of sequents closed under the following clauses:
(a) $A \vdash A$;
(b) $(B \backslash C) \vdash(A \backslash D)$ if $A \vdash B$ and $C \vdash D$;
(c) $(C / B) \vdash(D / A)$ if $A \vdash B$ and $C \vdash D$;
(d) $(A \bullet C) \vdash(B \bullet D)$ if $A \vdash B$ and $C \vdash D$;
(e) $B \vdash(A \backslash C)$ if and only if $(A \bullet B) \vdash C$;
(f) $A \vdash(C / B)$ if and only if $(A \bullet B) \vdash C$.

Proof. Let $S$ be the least set closed under the above conditions, and let $T$ be the set of tautologies of $\mathbf{N L}^{\backslash} \bullet \bullet$. We first note that Clauses $a, b, c, d, e$, and $f$ correspond to admissible rules of $\mathbf{N L} \backslash \bullet$. Therefore, $S \subset T$.

Then, to prove that $T \subset S$ consists in a routine induction on the length of the sequent proofs of $\mathbf{N L} \backslash \bullet \bullet$.

\section{The product-free case}

Proof search in the non-associative Lambek calculus takes advantage of the structure of the sequents. However, the reconstruction of a proof from a sequent is not as simple as it might seem at first sight. Indeed the backward application of the inference rules is not completely deterministic, as shown by the following derivations, which correspond to two different proofs of the same sequent.

$$
\begin{aligned}
& \frac{b \vdash b \quad a \vdash a}{\frac{b, b \backslash a \vdash a}{b \backslash a \vdash b \backslash a} \quad a \vdash a} \\
& \frac{\frac{a /(b \backslash a), b \backslash a \vdash a}{a /(b \backslash a) \vdash a /(b \backslash a)}}{a /(b \backslash a),(a /(b \backslash a)) \backslash a \vdash a} a \vdash a \\
& \frac{\frac{b \vdash b a}{b, b \backslash a \vdash a}}{\frac{b \vdash a /(b \backslash a)}{b, a} a} \quad a \\
& \frac{\frac{(a /(b \backslash a)) \backslash a \vdash b \backslash a}{a /(b \backslash a),(a /(b \backslash a)) \backslash a \vdash a}}{}
\end{aligned}
$$


Now it is easy to construct, from the above example, sequents with an exponential number of possible proofs. Consequently a brute force search based on the sequent calculus of Section 2 cannot be polynomial in time.

In the product free case, the polynomiality of the decision problem may be obtained as a consequence of the following key property: any derivation of a twoformula sequent may be transformed, by permuting the rules, into a derivation where each two-premise inference rule is immediately followed by a one-premise inference rule. Consequently, any derivation of a two-formula sequent may be transformed into a derivation whose sequents contain at most three formulas. This key property fails when the product is present. This is shown, for instance, by the following counterexample.

$$
\frac{\frac{a \vdash a \quad b \vdash b}{(a, b) \vdash a \bullet b \quad c \vdash c}}{\frac{((a, b),(a \bullet b) \backslash c) \vdash c}{(a, b) \vdash c /((a \bullet b) \backslash c)}} \frac{a \vdash(c /((a \bullet b) \backslash c)) / b}{a}
$$

In order to better understand the meaning of the key property, consider the two-premise rules of the sequent calculus of Section 2. Each of these rules introduces two connectives: an actual conjunctive connective, which is the active connective of the rule (i.e., a negative implication, or a positive product), and a possible disjunctive connective, which is introduced by the rule as a metaconnective (i.e., a comma). When deriving a two formula sequent, this metaconnective will be eventually turned into a positive implication or a negative product.

In the product-free case, the key property says that each comma may be turned into an actual connective as soon as it is introduced. Consequently, by merging the left and the right introduction rules, one obtains a complete system whose rules introduce two dual connectives at the same time:

$$
A \vdash A
$$

$$
\begin{aligned}
& \frac{A \vdash B \quad C \vdash D}{(B \backslash C) \vdash(A \backslash D)} \quad \frac{A \vdash B \quad C \vdash D}{A \vdash(D /(B \backslash C))} \\
& \frac{A \vdash B \quad C \vdash D}{(C / B) \vdash(D / A)} \quad \frac{A \vdash B \quad C \vdash D}{A \vdash((C / B) \backslash D)}
\end{aligned}
$$

In the case of $\mathbf{N L} \backslash / \bullet$, it is still possible to design such a system, where each rule introduces a pair of dual connectives. However, because of the failure of the key property, this system manipulates a notion of context. This is explained in the next section. 


\section{A calculus with contexts}

In this section, we define a context to be a formula with a hole (remark that this notion of context is different from the one of Section 2):

$\mathcal{C}[]::=[]|(\mathcal{C}[] \backslash \mathcal{F})|(\mathcal{F} \backslash \mathcal{C}[])|(\mathcal{C}[] / \mathcal{F})|(\mathcal{F} / \mathcal{C}[])|(\mathcal{C}[] \bullet \mathcal{F})|(\mathcal{F} \bullet \mathcal{C}[])$

We let $\Gamma[], \Delta[], \ldots$ range over contexts, and we write $\Gamma[A]$ to denote the formula obtained by filling the hole in $\Gamma[]$ with the formula $A$. We also say that a context $\Gamma[]$ is a correct positive (respectively, negative) context if and only if $A \vdash \Gamma[B]$ (respectively, $\Gamma[A] \vdash B$ ) is a tautology whenever $A \vdash B$ is. This notion of correctness is the keystone of the following calculus, which includes inference rules that allow correct contexts to be derived.

\section{Sequent rules}

$$
\begin{gathered}
A \vdash A \quad(\mathrm{Id}) \\
\frac{A \vdash B \quad C \vdash D}{(B \backslash C) \vdash(A \backslash D)}(\backslash) \quad \frac{A \vdash B \quad C \vdash D}{(C / B) \vdash(D / A)} \quad(/) \\
\frac{A \vdash B \quad C \vdash D}{(A \bullet C) \vdash(B \bullet D)}(\bullet) \\
\frac{A \vdash B \vdash_{N} \Gamma[]}{\Gamma[A] \vdash B}\left(\operatorname{Cont}_{N}\right) \quad \frac{A \vdash B \quad{ }_{P} \Gamma[]}{A \vdash \Gamma[B]} \quad\left(\operatorname{Cont}_{P}\right)
\end{gathered}
$$

Negative context rules

$$
\boldsymbol{\bullet}_{N}[] \quad([]-\mathrm{N})
$$

$\frac{A \vdash B \quad \vdash_{N} \Gamma[] \vdash_{N} \Delta[]}{\vdash_{N}(A \bullet \Gamma[(B \backslash \Delta[])])}(\bullet \backslash-\mathrm{N}) \quad \frac{A \vdash B \quad \vdash_{N} \Gamma[] \vdash_{N} \Delta[]}{\vdash_{N}(\Gamma[(\Delta[] / B)] \bullet A)}(\bullet /-\mathrm{N})$

\section{Positive context rules}

$$
\vdash_{P}[] \quad([]-\mathrm{P})
$$

$$
\frac{A \vdash B \quad \vdash_{P} \Gamma[] \quad{ }_{P} \Delta[]}{\vdash_{P}(A \backslash \Gamma[(B \bullet \Delta[])])}(\backslash \bullet-\mathrm{P}) \quad \frac{A \vdash B \quad \vdash_{P} \Gamma[] \vdash_{P} \Delta[]}{\vdash_{P}(\Gamma[(\Delta[] \bullet B)] / A)}(/ \bullet-\mathrm{P})
$$


$\frac{B \vdash A \quad \vdash_{N} \Gamma[] \quad \vdash_{P} \Delta[]}{\vdash_{P}(A / \Gamma[(\Delta[] \backslash B)])}(\bigwedge-\mathrm{P}) \quad \frac{B \vdash^{\prime} A \vdash_{N} \Gamma[] \quad \vdash_{P} \Delta[]}{\vdash_{P}(\Gamma[(B / \Delta[])] \backslash A)}(\backslash / \mathrm{P})$

We now prove that the above system, which we call SC, is a sound and complete axiomatisation of $\mathbf{N L} \backslash \cdot \bullet$.

Proposition 3. (Soundness) Let $A \vdash B$ be a sequent derivable according to system $S C$. Then $A \vdash B$ is a tautology of $\mathbf{N L}^{\backslash} \bullet$.

Proof. The proof is carried out by induction on the $S C$-derivation of $A \vdash B$. The cases of Axiom Id, Rules $\backslash, /$, and $\bullet$ are straightforward because they correspond, respectively, to Conditions $a, b c$ and $d$ of Proposition 2. Rules Cont ${ }_{N}$ and Cont $_{P}$ correspond to the definition of correctness for the contexts. Consequently, it remains to prove that the negative and positive context rules allow only correct contexts to be derived. We handle the case of the negative contexts and leave the other case, which is similar, to the reader.

Let $C \vdash D$ be a tautology of $\mathbf{N L} \backslash / \bullet$ and let $\Theta[]$ be a context such that $\vdash_{N} \Theta[]$ is derivable. We must prove that $\Theta[C] \vdash D$ is a tautology.

The case where $\Theta$ is obtained by axiom []- $N$ is obvious.

If $\Theta$ is obtained by Rule $\bullet-N$ then $\Theta=(A \bullet \Gamma[(B \backslash \Delta[])])$ where, by induction hypothesis, $A \vdash B$ is a tautology and $\Gamma[], \Delta[]$ are correct negative contexts. Then $\Delta[C] \vdash D$ is a tautology, and so is $(B \backslash \Delta[C]) \vdash(A \backslash D)$. Hence $\Gamma[(B \backslash$ $\Delta[C])] \vdash(A \backslash D)$ is also a tautology and, by Condition e of Proposition 2, so is $(A \bullet \Gamma[(B \backslash \Delta[C])]) \vdash D$.

The case where $\Theta$ is obtained by Rule $\bullet /-N$ is similar.

In order to prove the completeness, we first establish two lemmas.

Lemma 1. If $\vdash_{N} \Gamma[]$ and $\vdash_{N} \Delta[]$ are both derivable, so is $\vdash_{N} \Gamma[\Delta[]]$.

Proof. A straightforward induction on the derivation of $\vdash_{N} \Gamma[]$.

Lemma 2. If $\vdash_{P} \Gamma[]$ and $\vdash_{P} \Delta[]$ are both derivable, so is $\vdash_{P} \Gamma[\Delta[]]$.

Proof. A straightforward induction on the derivation of $\mathfrak{}_{P} \Gamma[]$.

We say that an SC-derivation is normal if the three following conditions hold:

(a) it is not the case that the right premise of any occurrence of Rule $\operatorname{Cont}_{N}$ (respectively, Rule $\operatorname{Cont}_{P}$ ) is obtained by the Axiom []-N (respectively, Axiom []-P);

(b) it is not the case that the left premise of any occurrence of Rule Cont ${ }_{N}$ (respectively, Rule $\operatorname{Cont}_{P}$ ) is obtained as the conclusion of another occurrence of Rule $\operatorname{Cont}_{N}$ (respectively, Rule $\operatorname{Cont}_{P}$ );

(c) Axiom Id is restricted to atomic formulas. 
Lemma 3. Any SC-derivation may be turned into a normal derivation.

Proof. The occurrences of Rule Cont N $_{\text {or Cont }}$ that do not satisfy Condition a are clearly useless. On the other hand, the occurrences of Rule Cont R $_{\text {and }}$ Cont $_{P}$ that do not satisfy Condition $b$ may be eliminated by Lemmas 1 and 2. Finally, Rules \, /, and $\bullet$ allow any tautology of the form $A \vdash A$ to be derived from axioms on atomic formulas.

Proposition 4. (Completeness) Let $A \vdash B$ be a tautology of $\mathbf{N L} \backslash \bullet$. Then $A \vdash B$ is derivable according to system $S C$.

Proof. We prove that the set of SC-derivable sequents is closed under the conditions of Proposition 2. This is clearly the case for Conditions a, b, c, d since they respectively correspond to Axiom Id and Rules $\backslash$, /, and $\bullet$. Therefore, it remains to prove that the set of $S C$-derivable sequents is closed under Conditions e and $f$. This amounts to proving that the following rules are admissible:

$$
\frac{(A \bullet B) \vdash C}{B \vdash(A \backslash C)}(e 1) \frac{B \vdash(A \backslash C)}{(A \bullet B) \vdash C}(e 2) \frac{(A \bullet B) \vdash C}{A \vdash(C / B)}(f 1) \frac{A \vdash(C / B)}{(A \bullet B) \vdash C} \text { (f2) }
$$

We show that each of these rules is admissible by performing a case analysis of the normal SC-derivations.

A. Admissibility of Rule e1.

A.1. The last rule of the $S C$-derivation is Rule $\bullet$ :

$$
\frac{A \vdash B \quad C \vdash D}{(A \bullet C) \vdash(B \bullet D)}(\bullet)
$$

The derivation may be transformed as follows:

$$
\frac{C \vdash D \frac{A \vdash B \quad{ }_{P}[] \vdash_{P}[]}{\vdash_{P}(A \backslash(B \bullet[]))}\left(C^{\circ} \text { ont }_{P}\right)}{C \vdash(A \backslash(B \bullet D))}
$$

A.2. The last rule of the SC-derivation is Rule Cont $_{N}$. We distinguish between two subcases.

A.2.1. The right premise of Rule Cont R is obtained by application of Rule $\bullet \backslash N$ :

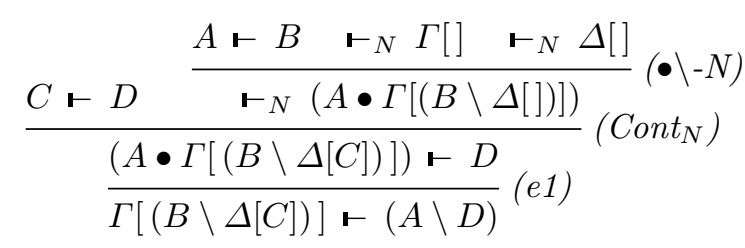


The derivation may be transformed as follows:

$$
\frac{A \vdash B \quad \frac{C \vdash D \quad{ }_{N} \Delta[]}{\Delta[C] \vdash D}\left(\text { Cont }_{N}\right)}{\frac{(B \backslash \Delta[C]) \vdash(A \backslash D)}{\Gamma}\left({ }^{\prime}{ }_{N} \Gamma[]\right.}\left(\text { Cont }_{N}\right)
$$

A.2.2. The right premise of Rule Cont ${ }_{N}$ is obtained by application of Rule $\bullet /-N$ :

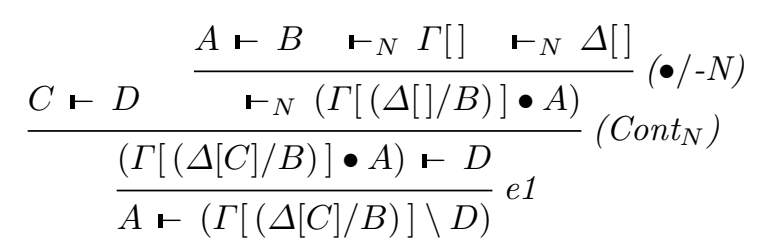

The derivation may be transformed as follows:

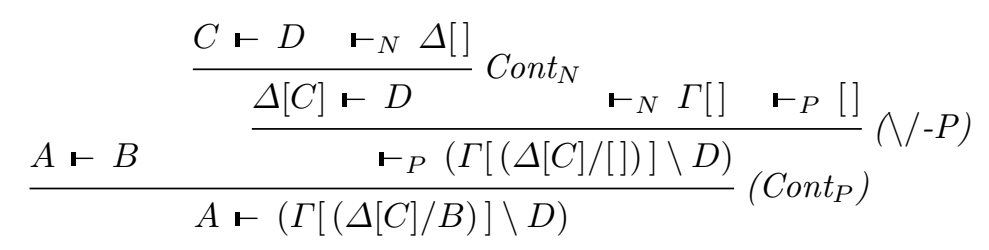

A.3. The last rule of the SC-derivation is Rule Cont $t_{P}$. Again, we distinguish between two subcases.

A.3.1. The left premise of Rule Cont $_{P}$ is obtained by applying Rule •

$$
\frac{\frac{A \vdash B \quad C \vdash D}{(A \bullet C) \vdash(B \bullet D)}(\bullet) \quad{ }_{P} \Gamma[]}{\frac{(A \bullet C) \vdash \Gamma[(B \bullet D)]}{C \vdash(A \backslash \Gamma[(B \bullet D)])}(e 1)}\left(\text { Cont }_{P}\right)
$$

The derivation may be transformed as follows:

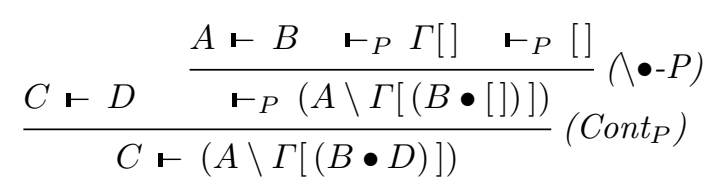

A.3.2. The left premise of Rule Cont P $_{P}$ is obtained by applying Rule Cont : $_{\text {: }}$

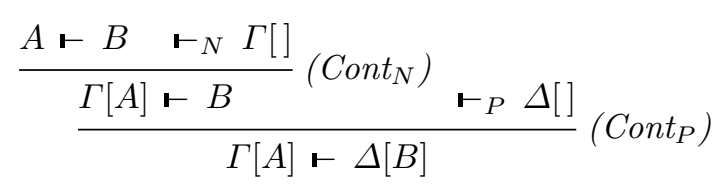


This case may be reduced to case A.2 by permuting the two rules as follows:

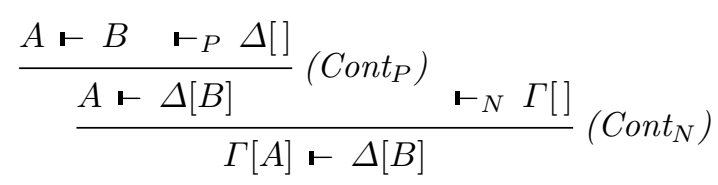

B. Admissibility of Rule e2.

B.1. The last rule of the $S C$-derivation is Rule $\backslash$ :

$$
\frac{A \vdash B \quad C \vdash D}{(B \backslash C) \vdash(A \backslash D)}(N)
$$

The derivation may be transformed as follows:

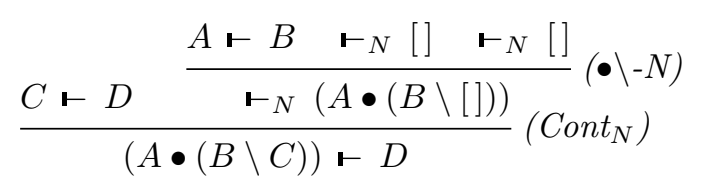

B.2. The last rule of the $S C$-derivation is Rule Cont $_{N}$. We distinguish between two subcases.

B.2.1. The left premise of Rule Cont ${ }_{N}$ is obtained by applying Rule $\backslash$ :

$$
\frac{\frac{A \vdash B \quad C \vdash D}{(B \backslash C) \vdash(A \backslash D)}() \quad{ }_{N} \Gamma[]}{\frac{\Gamma[(B \backslash C)] \vdash(A \backslash D)}{(A \bullet \Gamma[(B \backslash C)]) \vdash D}(\text { e } 2)}\left(\text { Cont }_{N}\right)
$$

The derivation may be transformed as follows:

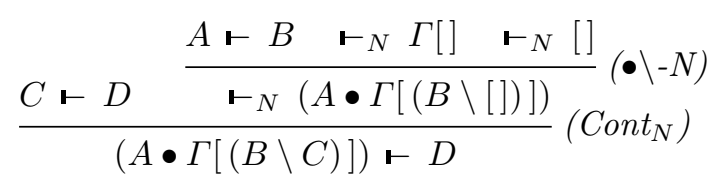

B.2.2. The left premise of Rule Cont ${ }_{N}$ is obtained by applying Rule Cont $t_{P}$ :

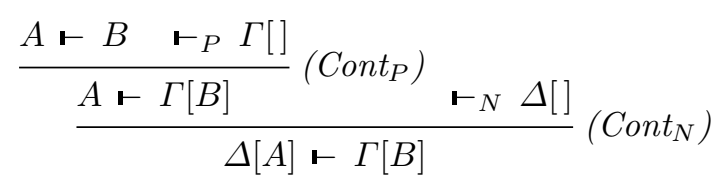

This case is reduced to case B.3 by permuting the two rules:

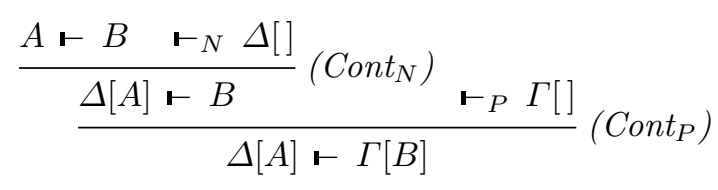


B.3. The last rule of the $S C$-derivation is Rule Cont $t_{P}$. There are two subcases.

B.3.1. The right premise of Rule Cont R $_{P}$ is obtained by application of Rule $\backslash \bullet-P$ :

$$
\frac{C \vdash D \quad \frac{A \vdash B \quad \vdash_{P} \Gamma[] \quad \vdash_{P} \Delta[]}{\vdash_{P}(A \backslash \Gamma[(B \bullet \Delta[])])}}{\frac{C \vdash(A \backslash \Gamma[(B \bullet \Delta[D])])}{(A \bullet C) \vdash \Gamma[(B \bullet \Delta[D])]}(\text { e2 })} \text { Cont } P_{P}
$$

The derivation may be transformed as follows:

$$
\frac{A \vdash B \quad \frac{C \vdash D \quad{ } \Delta[]}{C \vdash \Delta[D]}\left(\text { Cont }_{P}\right)}{\frac{(A \bullet C) \vdash(B \bullet \Delta[D])}{(A \bullet C) \vdash \Gamma[(B \bullet \Delta[D])]} \quad \vdash_{P} \Gamma[]}\left(\text { Cont }_{P}\right)
$$

B.3.1. The right premise of Rule Cont R $_{P}$ is obtained by application of Rule $\bigvee-P$ :

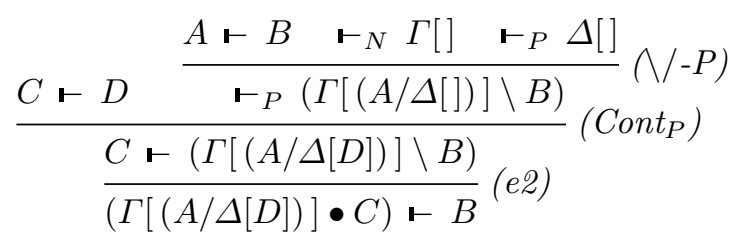

The derivation may be transformed as follows:

$$
\frac{A \vdash B \frac{\frac{C \vdash D \quad \vdash_{P} \Delta[]}{C \vdash \Delta[D]}\left(\text { Cont }_{P}\right) \vdash_{N} \Gamma[] \quad \vdash_{N}[]}{\vdash_{N}(\Gamma[([] / \Delta[D])] \bullet C)}\left(\text { Cont }_{N}\right)}{(\Gamma[(A / \Delta[D])] \bullet C) \vdash B}(\bullet)
$$

C. Admissibility of Rule f1. This part of the proof is symmetric to Part A.

D. Admissibility of Rule f2. This part of the proof is symmetric to Part B.

\section{Polynomiality}

Let $A$ be a formula and $\Gamma[]$ be a context. We say that $\Gamma[]$ is a subcontext of $A$ if and only if there exists a context $\Delta[]$ and a formula $B$ such that $A=\Delta[\Gamma[B]]$. Remark that if $A$ is a formula of length $n$ then the number of subformulas of $A$ is bounded by $n$, and the number of subcontexts of $A$ is bounded by $n^{2}$.

We immediately obtain the following property.

Lemma 4. The SC-derivations satisfy the subformula/subcontext property, i.e., all the formulas and contexts occurring in an $S C$-derivation are subformulas and subcontexts of the conclusion of this $S C$-derivation. 
Proof. A straightforward induction on SC-derivations.

From this lemma, we easily derive our main result.

Theorem 1. The non-associative Lambek calculus is decidable in polynomial time.

Proof. Let $A \vdash B$ be a two formula sequent of $\mathbf{N L} \backslash / \bullet$. By Propositions 3 and 4 , $A \vdash B$ is a tautology of $\mathbf{N L} \backslash \bullet \bullet$ if and only if there exist an $S C$-derivation of it. Now, by Lemma 4, any possible $S C$-derivation of $A \vdash B$ will be made up of two kinds of expressions:

- subcontexts of either $A$ or $B$,

- sequents of the form $C \vdash D$, where $C$ and $D$ are subformulas of $A$ or $B$.

The number of such expressions is bounded by $2 n^{2}$, where $n$ is the sum of the lengths of $A$ and $B$. Consequently, a brute force search algorithm for constructing a possible $S C$-derivation of $A \vdash B$ will terminate in polynomial time if its search space is organised as a DAG rather than as a tree.

Remark 1. Organizing the proof-search space in such a way that different possible proofs share the sub-proofs they have in common is needed in order to get a polynomial algorithm. Nevertheless, the bottum-up strategy suggested by the proof of Theorem 1 is not the only possible way. In practice, one could prefer top-down strategies, such as the so-called inverse method, that take advantage of dynamic programming techniques.

Acknowledgements I wish to thank Glyn Morrill, François Lamarche, JeanYves Marion and Adam Cichon for helpful discussions and comments.

\section{References}

1. E. Aarts and K. Trautwein. Non-associative lambek categorial grammar in polynomial time. Mathematical Logic Quaterly, 41:476-484, 1995.

2. J.-Y. Girard. Linear logic. Theoretical Computer Science, 50:1-102, 1987.

3. M. Kanovich. Horn programming in linear logic is np-complete. In 7-th annual IEEE Symposium on Logic in Computer Science, pages 200-210. IEEE Computer Society Press, 1992.

4. J. Lambek. The mathematics of sentence structure. Amer. Math. Monthly, 65:154$170,1958$.

5. J. Lambek. On the calculus of syntactic types. In Studies of Language and its Mathematical Aspects, pages 166-178, Providence, 1961. Proc. of the 12th Symp. Appl. Math..

6. M. Moortgat. Categorial type logic. In J. van Benthem and A. ter Meulen, editors, Handbook of Logic and Language, chapter 2. Elsevier, 1997. 